\title{
Lymphovascular and Perineural Invasions Are Independently Associated with Advanced Colorectal Carcinoma
}

\author{
Hossein Yahyazadeh (iD ${ }^{1}$, Ahmad R Mafi (iD) ${ }^{2}$, Elham Khatooni (iD ${ }^{3}$, Marzieh Beheshti (iD) ${ }^{1,}$ and Azita \\ Abdollahinejad (iD) 1 \\ ${ }^{1}$ Clinical Cancer Research Center, Milad General Hospital, Tehran, Iran \\ ${ }^{2}$ Shahid Beheshti University of Medical Sciences, Tehran, Iran \\ ${ }^{3}$ Department of Epidemiology and Biostatistics, School of Public Health, Tehran University of Medical Sciences, Tehran, Iran \\ Corresponding author: Clinical Cancer Research Center, 12th Floor, Milad General Hospital, Hemat Highway, Tehran, Iran. Tel/Fax : +98-2188621026, Email: \\ m.beheshtee1987@yahoo.com
}

Received 2019 June 20; Revised 2019 September 05; Accepted 2019 September 08.

\begin{abstract}
Background: Lymphovascular invasion (LVI) and perineural invasion (PNI) are relatively common in various malignancies including colorectal cancers and have been shown to have prognostic significance.

Objectives: The aim of this study was to identify the clinical and pathological variables associated with LVI and PNI in patients with colorectal carcinoma, who have been treated at Milad General Hospital in Tehran, Iran.

Methods: The records of the patients with the diagnosis of colorectal carcinoma, who had undergone an operation at Milad General Hospital (Tehran, Iran) between 2012 and 2017, were reviewed. All patients, whose pathology reports and treatment records were available at Milad Hospital, were included. Relevant demographic, pathological, and surgical data, including age, gender, tumor location, maximum tumor size, pathologic Tumor, Node, Metastasis (TNM) stage, and grade and number of removed lymph nodes were extracted from the medical records.

Results: In total, 547 patients ( 374 cases of colon cancer and 173 cases of rectal cancer) enrolled in the study. The prevalence of LVI and PNI was $16.4 \%$ and 30.7\%, respectively. LVI and PNI were found to be associated with higher tumor grade, higher T-stage, and higher overall stage.

Conclusions: Colorectal carcinomas with positive LVI or PNI are more likely to have a higher grade, higher T-stage, and higher overall stage, and PNI is an independent factor for advanced disease.
\end{abstract}

Keywords: Colorectal Cancer, Lymphovascular Invasion, Perineural Invasion

\section{Background}

The incidence of colorectal cancer, as one of the leading causes of cancer-related mortality worldwide, has been increasing over the last 25 years in Iran. Following cancers of breast and stomach, it is the third most common cancer among Iranian people with the annual incidence of more than 7100 cases (1).

Lymphovascular invasion (extension of tumor cells into lymphatic and/or blood vessels, LVI) and perineural invasion (neoplastic invasion of nerves and nerve sheath, $\mathrm{PNI}$ ) are relatively common in various malignancies including colorectal cancers and have been shown to have prognostic significance.

\section{Objectives}

The aim of this study was to identify the clinical and pathological variables associated with LVI and PNI in colorectal cancer.

\section{Methods}

In this observational cross-sectional study, the records of patients with the diagnosis of colorectal, who had undergone an operation at Milad General Hospital (Tehran, Iran) between 2012 and 2017, were reviewed. All patients, whose pathology reports and treatment records were available at Milad Hospital, were included in the study (all available cases) and cases with incomplete records and missing necessary data were excluded from the study.

Relevant demographic, pathological, and surgical data, including age, gender, tumor location, maximum 
tumor size, pathologic Tumor, Node, Metastasis (TNM) stage, and grade and number of removed lymph nodes were extracted from medical records.

Tumor stages were defined according to the 7th edition of American Joint Committee on Cancer (AJCC) TNM staging system as follows: T1: tumor invades submucosa; T2: tumor invades muscularis propria; T3: tumor invades through the muscularis propria into the pericolorectal tissues; T4: tumor invades through the visceral peritoneum or directly invades or adheres to other adjacent organs or structures (2). Tumor grading was assessed according to the World Health Organization guidelines (3). This study was approved by the Ethics Committee of Milad Hospital.

\subsection{Statistical Analysis}

The chi-square test was used to evaluate the primary association among independent variables with LVI ( $0=$ No, 1 $=$ Yes $)$ and PNI ( 0 = No, $1=$ Yes $)$. Adjusted binary logistic regression models were utilized to test the effect of the independent variables on both LVI and PNI in separate models. Afterward, a mixed variable of LVI and PNI was built by 3 categories of $0=$ subjects, who had no LVI and PNI, $1=$ subjects, who had at least one of them, and 2 = subject, who had both PNI and LVI. Multinomial logistic regression models were conducted to examine the association between independent variables and mixed variables. Due to the small sample size, $\mathrm{T} 1$ cases in binary logistic regression and $\mathrm{T} 1$, $\mathrm{T} 2$, and stage I cases in multinomial regression were excluded from the analysis. In the modeling process, the first group of dependent variables was considered as the reference except for the AJCC stage. Statistical analysis was performed, using SPSS version 23 (IBM Corp. Released 2015. IBM SPSS Statistics for Windows, version 23.0. Armonk, NY: IBM Corp). P values of $\leq 0.05$ were considered as statistically significant.

\section{Results}

In total, 547 patients ( 374 cases of colon cancer and 173 cases of rectal cancer) enrolled in the study. The results of the study are shown in Tables 1 to 3.

The prevalence of LVI and PNI in our study was $16.4 \%$ and $30.7 \%$, respectively. There was no difference between the incidence of LVI+ or PNI+ in different genders in either colon or rectal cancer. Although there was a trend toward more cases of LVI+ in the right side of the colon compared to the left side and rectum, there was no statistically significant difference between LVI+ cases in the colon (17.4\%) and rectum $(14.5 \%)(\mathrm{P}=0.35)$.

Patients with positive LVI were significantly more likely to have a higher T-stage $(\mathrm{P}=0.01)$, tumor size of $\geq 5 \mathrm{~cm}(\mathrm{P}=$ 0.01), higher grade, and higher overall AJCC stage $(\mathrm{P}<0.01$ and $\mathrm{P}<0.01$, respectively). In the same way, patients with positive PNI were more likely to have higher T-stage, higher grade, and higher overall stage $(\mathrm{P}<0.01, \mathrm{P}<0.01$, and $\mathrm{P}<$ 0.01 , respectively).

Using binary logistic regression, it was found that patients with LVI+ were more likely to have grade II or III diseases $(\mathrm{OR}=23.87, \mathrm{CI}=8.49-67.07$; and $\mathrm{OR}=2.05, \mathrm{CI}=1.02$ 4.13). Patients aged 60 years old and over were more likely to have LVI than the reference group $(\mathrm{CI}=0.98$ - 3.22). Furthermore, binary logistic regression confirmed that PNI+ remained statistically significant for histological grade II and III and T3 compared with their reference group.

Using the adjusted multinomial logistic regression analysis, we found that the odds of having at least one of the LVI or PNI in grade II and III were significantly higher than the reference group $(\mathrm{OR}=2.17, \mathrm{CI} 95 \%=1.28-3.67$; and $\mathrm{OR}=8.54, \mathrm{CI} 95 \%=2.70-26.94)$ and it was also true when having both PNI and LVI $(\mathrm{OR}=5.87, \mathrm{CI} 95 \%=2.23-15.46$; and $\mathrm{OR}=119.66, \mathrm{CI}=28.42-503.80)$.

The chance of having at least one or both LVI and PNI in stage III was higher than the reference group $(\mathrm{OR}=3.44$, $\mathrm{CI}=2.06$ - 5.73; and $\mathrm{OR}=73.38, \mathrm{CI}=19.21$ - 280.16) and the same was found for having at least one or both LVI and PNI in stage $\mathrm{II}(\mathrm{OR}=5.20, \mathrm{CI}=1.59-16.99$; and $\mathrm{OR}=16.18, \mathrm{CI}=6.24$ - 41.92).

Unadjusted multinomial logistic regression showed that the chance of having both LVI and PNI compared with the reference group was significantly higher for patients of $\geq 60$ years old, grade II and III, tumor size of $\geq 5 \mathrm{~cm}$, and stage II and III.

\section{Discussion}

The results of the present study showed that patients with LVI+ were more likely to have higher tumor grade, higher T-stage, larger tumor size, and higher overall stage of colorectal carcinoma. These results are in line with the results of many previous studies (4-6). We also found that the presence of PNI, regardless of the status of LVI, is associated with higher tumor grade, higher T-stage, and higher overall stage.

While some studies have demonstrated that LVI+ is not associated with age (4, 6-8) or is associated with younger age (9), in the current study, patients aged 60 years old and over were more likely to have LVI or PNI, which is similar to the results of Lim SB et al.'s study (10).

Similar to many previous studies, this study showed that LVI or PNI are not associated with gender and age (4, 6-8), while in Al-Sukhni et al.'s study, male gender was associated with more incidence of PNI (5).

For years, the prognostic significance of LVI in colorectal cancer was a subject of controversy. While many studies had considered its presence as a negative prognostic 
Yahyazadeh $\mathrm{H}$ et al.

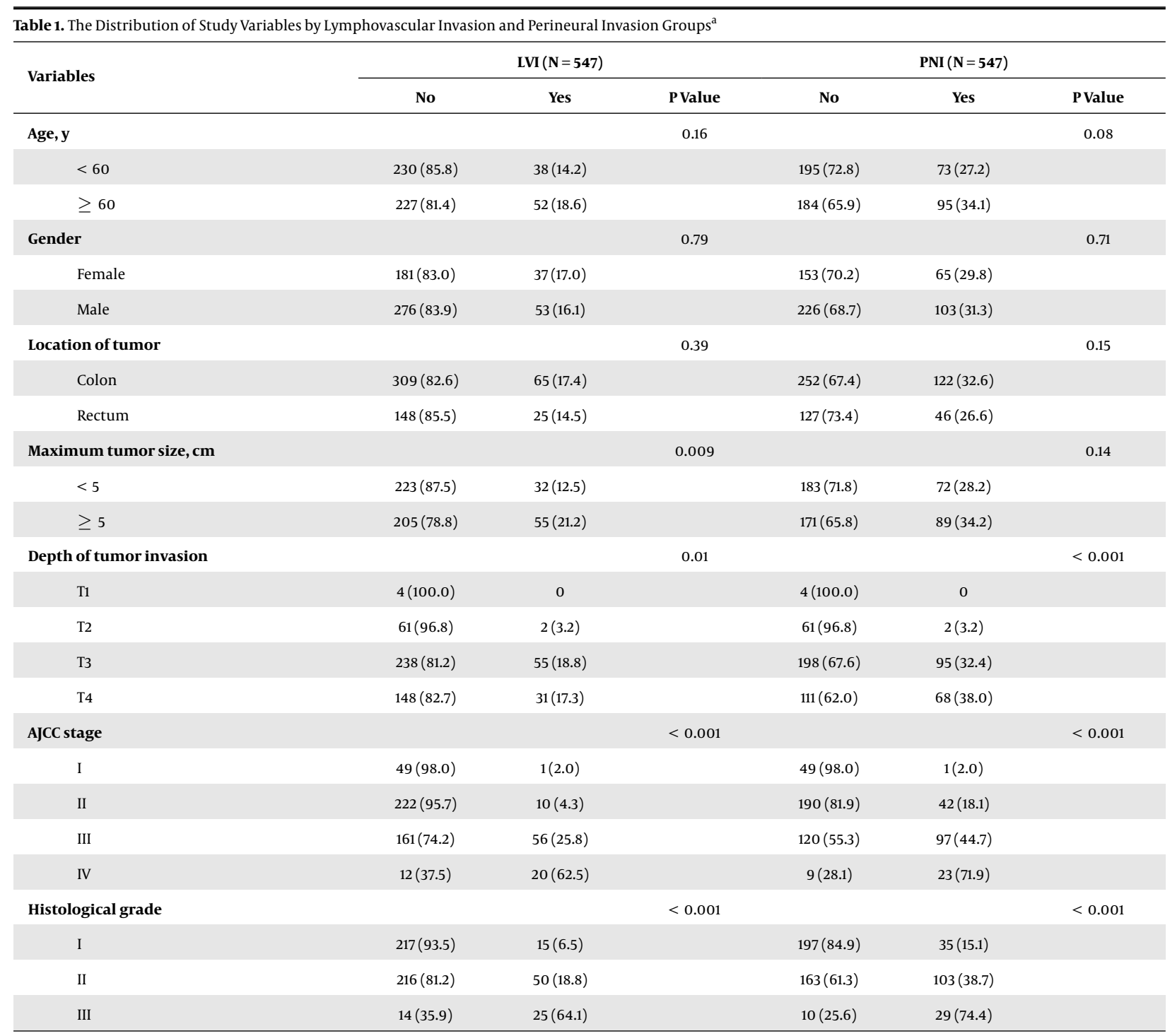

Abbreviations: AJCC, American Joint Committee on Cancer; LVI, lymphovascular invasion; PNI, perineural invasion. ${ }^{\mathrm{a}}$ Data presented as frequency (\%).

factor, some studies had demonstrated that LVI was of no prognostic significance for colorectal cancers (10). Recent studies have resolved this controversy to a great extent, as several researchers have shown that the presence of LVI in colorectal cancer is a strong stage-independent prognostic factor. LVI is now thought to be involved in the development of lymphatic metastasis and it is considered by many international guidelines as an independent indicator of progressive disease, which can negatively affect patients' survival $(11,12)$. Based on the College of American Pathologists Consensus Statement, LVI is considered as a Category I factor, which has been definitively proven to be of prognostic importance based on evidence from multi- ple statistically robust published trials (13).

PNI refers to the process of neoplastic invasion of nerves and nerve sheath. In many malignancies, including cancers of head and neck, pancreas, colon, and rectum, PNI is a marker of decreased survival and poor outcome (7). PNI is a distinct pathologic entity that can be present in the absence of LVI.

Several studies have demonstrated that the presence of PNI in colorectal tumoral cells is associated with higher rates of locoregional recurrence and decreased survival (14-16).

In the literature, the prevalence of LVI and PNI has been reported to be $21 \%$ to $25 \%$ and $9.9 \%$ to $14 \%$ for LVI and PNI, 


\begin{tabular}{|c|c|c|c|c|}
\hline \multirow{2}{*}{ Variables } & \multicolumn{2}{|c|}{ LVI } & \multicolumn{2}{|c|}{ PNI } \\
\hline & OR $(95 \% \mathrm{CI})$ & PValue & $\mathrm{OR}(95 \% \mathrm{CI})$ & PValue \\
\hline \multicolumn{5}{|l|}{ Age, $y$} \\
\hline \multicolumn{5}{|c|}{$<60$ (ref) } \\
\hline$\geq 60$ & $1.77(0.98-3.22)$ & 0.057 & $1.49(0.94-2.35)$ & 0.08 \\
\hline \multicolumn{5}{|l|}{ Gender } \\
\hline \multicolumn{5}{|c|}{ Female (ref) } \\
\hline Male & $0.62(0.34-1.13)$ & 0.11 & $1.11(0.70-1.76)$ & 0.64 \\
\hline \multicolumn{5}{|c|}{ Location of tumor } \\
\hline \multicolumn{5}{|c|}{ Colon (ref) } \\
\hline Rectum & $1.19(0.62-2.25)$ & 0.59 & $1.19(0.72-1.96)$ & 0.49 \\
\hline \multicolumn{5}{|c|}{ Maximum tumor size, $\mathrm{cm}$} \\
\hline \multicolumn{5}{|l|}{$<5$ (ref) } \\
\hline$\geq 5$ & $1.61(0.88-2.94)$ & 0.11 & $0.96(0.60-1.52)$ & 0.86 \\
\hline \multicolumn{5}{|c|}{ Depth of tumor invasion } \\
\hline \multicolumn{5}{|l|}{ T2 (ref) } \\
\hline T3 & $0.36(0.04-3.13)$ & 0.36 & $0.08(0.01-0.66)$ & 0.02 \\
\hline $\mathrm{T} 4$ & $1.42(0.76-2.67)$ & 0.27 & $0.79(0.49-1.27)$ & 0.34 \\
\hline \multicolumn{5}{|l|}{ AJCC stage } \\
\hline \multicolumn{5}{|l|}{ IV (ref) } \\
\hline III & $0.39(0.01-8.02)$ & 0.54 & $0.70(0.03-13.0)$ & 0.81 \\
\hline II & $3.64(0.19-68.14)$ & 0.38 & $2.93(0.16-52.25)$ & 0.46 \\
\hline I & $13.81(0.67-283.68)$ & 0.08 & $6.32(0.32-124.55)$ & 0.22 \\
\hline \multicolumn{5}{|c|}{ Histological grade } \\
\hline \multicolumn{5}{|l|}{ I (ref) } \\
\hline II & $2.05(1.02-4.13)$ & 0.04 & $3.21(1.93-5.34)$ & $<0.001$ \\
\hline III & $23.87(8.49-67.07)$ & $<0.001$ & $14.05(5.64-34.95)$ & $<0.001$ \\
\hline
\end{tabular}

Abbreviations: AJCC, American Joint Committee on Cancer; LVI, lymphovascular invasion; PNI, perineural invasion.

respectively (5). However, it is worth noting that studies that specifically reviewed the pathology samples for the presence of these features have reported higher rates of LVI and PNI positivity (33\% and 22\%, respectively) (5). By reevaluating the pathology slides of 50 patients with colorectal cancer, Harris et al. (12) found significant inter-observer variability in the diagnosis of LVI on hematoxylin and eosin (H \& E) slides, which were worse in cases of large vessel invasion and did not improve by immunohistochemistry. Another study by reviewing 381 pathology samples showed that the detection of vascular invasion was related to the quality of pathology assessments such as the number of assessed tissue blocks (17). These results highlight the need for high-quality pathology reporting, more accurate criteria, and standardized quality control for the evaluation of
LVI and PNI as the presence of these features can change the course of clinical treatment.

In the present study, $16.4 \%$ and $30.7 \%$ of cases were found to be positive for LVI and PNI, respectively. While the prevalence of LVI+ in our samples was similar to that of the previous studies, we had a higher rate of PNI+ compared to similar studies, for which we have no explanation.

In conclusion, in this study, we assessed the clinical and pathological variables associated with LVI and PNI in patients with colorectal carcinoma, who have been treated at a referral general hospital in Tehran, Iran. The results showed that colorectal carcinomas with positive LVI or PNI are more likely to have a higher grade, higher T-stage, and higher overall stage, and PNI is an independent factor for advanced disease. 


\begin{tabular}{|c|c|c|c|c|}
\hline \multirow{2}{*}{ Variables } & \multicolumn{2}{|c|}{ Unadjusted, OR(95\% CI) } & \multicolumn{2}{|c|}{ Adjusted, OR(95\% CI) } \\
\hline & At Least One & Both & At Least One & Both \\
\hline \multicolumn{5}{|l|}{ Age, y } \\
\hline \multicolumn{5}{|c|}{$<60$ (ref) } \\
\hline$\geq 60$ & $1.08(0.70-1.65)$ & $1.68^{\mathrm{a}}(1.0-2.81)$ & $1.15(0.70-1.90)$ & $2.27^{\mathrm{a}}(1.12-4.59)$ \\
\hline \multicolumn{5}{|l|}{ Gender } \\
\hline \multicolumn{5}{|c|}{ Female (ref) } \\
\hline Male & $1.34(0.86-2.09)$ & $0.88(0.53-1.47)$ & $1.49(0.89-2.51)$ & $0.59(0.29-1.19)$ \\
\hline \multicolumn{5}{|c|}{ Location of tumor } \\
\hline \multicolumn{5}{|c|}{ Colon (ref) } \\
\hline Rectum & $0.99(0.63-1.55)$ & $1.61(0.89-2.89)$ & $0.89(0.52-1.53)$ & $1.42(0.65-3.08)$ \\
\hline \multicolumn{5}{|c|}{ Maximum tumor size, $\mathrm{cm}$} \\
\hline \multicolumn{5}{|l|}{$<5$ (ref) } \\
\hline$\geq 5$ & $1.24(0.80-1.92)$ & $1.81^{\mathrm{a}}(1.07-3.06)$ & $1.08(0.65-1.78)$ & $1.55(0.76-3.17)$ \\
\hline \multicolumn{5}{|c|}{ Depth of tumor invasion } \\
\hline \multicolumn{5}{|l|}{ T3 (ref) } \\
\hline $\mathrm{T} 4$ & $0.89(0.57-1.40)$ & $0.87(0.51-1.49)$ & $0.85(0.51-1.42)$ & $1.10(0.54-2.25)$ \\
\hline \multicolumn{5}{|l|}{ AJCC stage } \\
\hline \multicolumn{5}{|l|}{ IV (ref) } \\
\hline III & $2.96^{\mathrm{b}}(1.86-4.71)$ & $10.88^{\mathrm{b}}(4.73-25.0)$ & $3.44^{\mathrm{b}}(2.06-5.73)$ & $73.38^{\mathrm{b}}(19.21-280.16)$ \\
\hline II & $4.92^{\mathrm{b}}(1.63-14.84)$ & $68.69^{\mathrm{b}}(21.66-217.79)$ & $5.20^{\mathrm{b}}(1.59-16.99)$ & $16.18^{\mathrm{b}}(6.24-41.92)$ \\
\hline \multicolumn{5}{|l|}{ Grade } \\
\hline \multicolumn{5}{|l|}{$\mathrm{I}(\mathrm{ref})$} \\
\hline II & $2.40^{\mathrm{b}}(1.50-3.81)$ & $6.54^{\mathrm{b}}(2.99-14.33)$ & $2.17^{\mathrm{b}}(1.28-3.67)$ & $5.87^{\mathrm{b}}(2.23-15.46)$ \\
\hline III & $7.98^{\mathrm{b}}(2.84-22.41)$ & $\begin{array}{c}74.64^{\mathrm{b}}(24.69- \\
225.64)\end{array}$ & $8.54^{\mathrm{b}}(2.70-26.94)$ & $\begin{array}{c}119.66^{\mathrm{b}}(28.42- \\
503.80)\end{array}$ \\
\hline
\end{tabular}

Abbreviation: AJCC, American Joint Committee on Cancer.

${ }^{\mathrm{a}} \mathrm{P}<0.05$

${ }^{\mathrm{b}} \mathrm{P}<0.01$.

\section{Acknowledgments}

The authors would like to thank the Cancer Research Center of Milad General Hospital (affiliated to the Iranian Social Security Organization) for their help and support.

\section{Footnotes}

Authors' Contribution: Study concept and design: Hossein Yahyazadeh; acquisition of data: Marzieh Beheshti and Azita Abdollahinejad; analysis and interpretation of data: Elham Khatooni, Ahmad R Mafi, and Hossein Yahyazadeh; drafting of the manuscript: Ahmad R Mafi, Hossein Yahyazadeh, Marzieh Beheshti, Azita Abdollahinejad, and Elham Khatooni; critical revision of the manuscript for important intellectual content: Hossein Yahyazadeh, Ahmad R Mafi, and Elham Khatooni; statistical analysis: Elham Khatooni; administrative, technical, and material support: Marzieh Beheshti and Azita Abdollahinejad; study supervision: Hossein Yahyazadeh and Ahmad R Mafi.

Conflict of Interests: The authors disclosed no conflict of interest.

Ethical Approval: This study was approved by the Ethics Committee of Milad Hospital.

Funding/Support: This study was supported by Milad General Hospital (affiliated to Iranian Social Security Organization). 


\section{References}

1. Khosravi Shadmani F, Ayubi E, Khazaei S, Sani M, Mansouri Hanis S, Khazaei S, et al. Geographic distribution of the incidence of colorectal cancer in Iran: A population-based study. Epidemiol Health. 2017;39. e2017020. doi: 10.4178/epih.e2017020. [PubMed: 28774167]. [PubMed Central: PMC5543296].

2. Sobin LH, Gospodarowicz MK, Wittekind C. TNM classification of malignant tumors. 7th ed. New York: Wiley; 2009.

3. Hamilton S, Aaltonen L. Tumours of the digestive system World Health Organization classification of tumours. Lyon: IARC. 2000;314:105-19.

4. Tsai HL, Huang CW, Chen CW, Yeh YS, Ma CJ, Wang JY. Survival in resected stage II colorectal cancer is dependent on tumor depth, vascular invasion, postoperative CEA level, and the number of examined lymph nodes. World J Surg. 2016;40(4):1002-9. doi: 10.1007/s00268015-3331-y. [PubMed: 26560149].

5. Al-Sukhni E, Attwood K, Gabriel EM, LeVea CM, Kanehira K, Nurkin SJ. Lymphovascular and perineural invasion are associated with poor prognostic features and outcomes in colorectal cancer: A retrospective cohort study. Int J Surg. 2017;37:42-9. doi:10.1016/j.ijsu.2016.08.528. [PubMed: 27600906].

6. Aktekin A, Ozkara S, Gurleyik G, Odabasi M, Muftuoglu T, Saglam A. The factors effecting lymphovascular invasion in adenocarcinoma of the colon and rectum. Indian J Surg. 2015;77(Suppl 2):314-8. doi: 10.1007/s12262-013-0816-5. [PubMed: 26730017]. [PubMed Central: PMC4692880].

7. Liebig C, Ayala G, Wilks JA, Berger DH, Albo D. Perineural invasion in cancer: A review of the literature. Cancer. 2009;115(15):3379-91. doi: 10.1002/cncr.24396. [PubMed:19484787].

8. Okamoto Y, Mitomi H, Ichikawa K, Tomita S, Fujimori T, Igarashi Y, et al. Effect of skip lymphovascular invasion on hepatic metastasis in colorectal carcinomas. Int J Clin Oncol. 2015;20(4):761-6. doi: 10.1007/s10147-014-0778-z. [PubMed: 25483315].

9. Inoki K, Sakamoto T, Takamaru H, Sekiguchi M, Yamada M, Nakajima T, et al. Predictive relevance of lymphovascular invasion in T1 colorectal cancer before endoscopic treatment. Endosc Int Open. 2017;5(12):E1278-
83. doi: 10.1055/s-0043-117952. [PubMed: 29218320]. [PubMed Central: PMC5718905].

10. Lim SB, Yu CS, Jang SJ, Kim TW, Kim JH, Kim JC. Prognostic significance of lymphovascular invasion in sporadic colorectal cancer. Dis Colon Rectum. 2010;53(4):377-84. doi: 10.1007/DCR.0b013e3181cf8ae5. [PubMed: 20305435].

11. Yuan H, Dong Q, Zheng B, Hu X, Xu JB, Tu S. Lymphovascular invasion is a high risk factor for stage I/II colorectal cancer: A systematic review and meta-analysis. Oncotarget. 2017;8(28):46565-79. doi: 10.18632/oncotarget.15425. [PubMed: 28430621]. [PubMed Central: PMC5542293].

12. Harris EI, Lewin DN, Wang HL, Lauwers GY, Srivastava A, Shyr Y, et al. Lymphovascular invasion in colorectal cancer: An interobserver variability study. Am J Surg Pathol. 2008;32(12):1816-21. doi: 10.1097/PAS.0b013e3181816083. [PubMed: 18779725]. [PubMed Central: PMC2605104].

13. Compton CC, Fielding LP, Burgart LJ, Conley B, Cooper HS, Hamilton SR, et al. Prognostic factors in colorectal cancer College of American Pathologists Consensus Statement 1999. Arch Pathol Lab Med. 2000;124(7):979-94. doi: 10.1043/00039985(2000)124<0979:PFICC>2.0.CO;2. [PubMed: 10888773].

14. Cienfuegos JA, Martinez P, Baixauli J, Beorlegui C, Rosenstone S, Sola JJ, et al. Perineural invasion is a major prognostic and predictive factor of response to adjuvant chemotherapy in stage I-II colon cancer. Ann Surg Oncol. 2017;24(4):1077-84. doi: 10.1245/s10434-016-5561-0. [PubMed: 27624582].

15. Huh JW, Kim HR, Kim YJ. Prognostic value of perineural invasion in patients with stage II colorectal cancer. Ann Surg Oncol. 2010;17(8):206672. doi: 10.1245/s10434-010-0982-7. [PubMed: 20182809].

16. Knijn N, Mogk SC, Teerenstra S, Simmer F, Nagtegaal ID. Perineural invasion is a strong prognostic factor in colorectal cancer: A systematic review. Am J Surg Pathol. 2016;40(1):103-12. doi: 10.1097/PAS.0000000000000518. [PubMed: 26426380].

17. Betge J, Pollheimer MJ, Lindtner RA, Kornprat P, Schlemmer A, Rehak $P$, et al. Intramural and extramural vascular invasion in colorectal cancer: Prognostic significance and quality of pathology reporting. Cancer. 2012;118(3):628-38. doi: 10.1002/cncr.26310. [PubMed: 21751188]. 